\title{
Predictors of Seronegative Conversion After Centralized Management of Syphilis Patients in Shenzhen, China
}

\section{OPEN ACCESS}

Edited by:

Joseph D. Tucker,

University of North Carolina at Chapel Hill, United States

Reviewed by:

Ngai Sze Wong,

The Chinese University of Hong Kong, Hong Kong SAR, China

Gifty Marley,

University of North Carolina China-Project (SESH Global), China

Weibin Cheng,

Guangdong Second Provincial

General Hospital, China

*Correspondence:

$\mathrm{Bo} \mathrm{Li}$

279805496@qq.com

Jinsong Mou

moujs@foxmail.com

†These authors have contributed equally to this work

Specialty section:

This article was submitted to Infectious Diseases - Surveillance,

Prevention and Treatment,

a section of the journal

Frontiers in Public Health

Received: 07 August 2021 Accepted: 03 November 2021 Published: 25 November 2021

Citation:

Luo Z, Ding Y, Yuan J, Wu Q, Tian L,

Zhang L, Li B and Mou J (2021)

Predictors of Seronegative Conversion

After Centralized Management of

Syphilis Patients in Shenzhen, China.

Front. Public Health 9:755037.

doi: 10.3389/fpubh.2021.755037

\section{Zhenzhou Luo ${ }^{1 \dagger}$, Yi Ding ${ }^{1+}$, Jun Yuan ${ }^{1}$, Qiuhong Wu ${ }^{1}$, Lishan Tian ${ }^{1}$, Li Zhang ${ }^{1}$, Bo Li ${ }^{1 *}$ and Jinsong $\mathrm{Mou}^{2 *}$}

${ }^{1}$ Shenzhen Nanshan Center for Chronic Disease Control, Shenzhen, China, ${ }^{2}$ Pingshan District Maternal and Child Healthcare Hospital of Shenzhen, Pingshan General Hospital of Southern Medical University, Shenzhen, China

Objective: The aim of this study was to explore the seronegative conversion status of syphilis patients after centralized management and to analyze potential determinants.

Materials and Methods: A retrospective population-based cohort study was conducted, and data for individuals who had been diagnosed with syphilis between 2011 and 2019 were retrieved from the Shenzhen Nanshan Center for Chronic Disease Control. Seroconversion statuses were summarized as percentages. Univariable and multiple Cox proportional hazard regression models were used to analyze the factors associated with seronegative conversion among syphilis patients.

Results: During the study period, 1,545 patients with syphilis participated in the syphilis convergence case management program on a voluntary basis, of whom 290 were excluded due to missing follow-up data. A total of $27.6 \%$ (346/1255) of patients with syphilis showed seronegative conversion. Multivariable analysis revealed that the following significantly determined syphilis seroconversion from positive to negative: younger age (15-19 years vs. $\geq 30$ years: $H R=2.18)$, male gender $(H R=1.45)$, lower baseline toluidine red unheated serum test (TRUST) titer of $\leq 1: 8(H R=2.23)$, and different disease stages, including latent syphilis $(H R=1.98)$, primary syphilis $(H R=7.67)$, and secondary syphilis $(H R=4.83)$.

Conclusions: Few patients with syphilis tested negative after treatment at the end of the study. Seronegative conversion in the patients was associated with age, sex, baseline TRUST titer, and syphilis stage.

Keywords: syphilis, seroconversion, Shenzhen, China, multivariable analysis, TRUST titer, centralized management, STIs

\section{INTRODUCTION}

Syphilis is a sexually transmitted infection (STI) caused by Treponema pallidum and is spread through contact with infectious lesions or body fluids (1). Patients typically develop cutaneous manifestations such as genital ulcers and various complications, including neurologic, renal, gastrointestinal, and hepatic diseases (2). 
Despite the availability of inexpensive and effective antibiotic therapy, syphilis remains a prevalent disease in developing countries and has re-emerged as a public health threat in developed nations. Syphilis has an estimated global prevalence of 36 million cases and an incidence of over 11 million cases annually (3). In the South African adult (15-49 years) population in 2017, the estimated prevalence of syphilis among women and men was $0.50 \%$ (95\% CI: $0.32-0.80 \%)$ and $0.97 \%(0.19-2.28 \%)$, respectively (4). In the United States, from 2013 to 2017, the national annual rate of reported primary and secondary (P\&S) syphilis cases increased by $72.7 \%$, from 5.5 to 9.5 cases per 100,000 individuals (5). A review of syphilis studies in Eastern Europe showed that even though the incidence was generally declining, high prevalence was reported in key populations, particularly sex workers and users of injectable drugs (6). In China, a range of unique biological and social forces, such as depletion of individuals with immunity, income gaps and a cultural climate that favors the re-emergence of sex work, are driving the spread of syphilis (7). With the current economic and social developments, the prevalence of sexually transmitted disease (STD) remains an important concern.

To control the disease, China has continually implemented massive syphilis control programs. However, during the past 20 years, syphilis has resurged in China (7), with a significant increase in incidence from 2003 to $2013(8,9)$. The syphilis burden has been greatest in coastal urban China areas, such as Guangdong Province (10). A meta-analysis showed that Guangdong was among the provinces of China with a very high syphilis prevalence of more than $10 \%$ (11). A significant rise in syphilis cases has been observed in Guangdong Province over the last decade (12). From January 2014 through June 2015, 82,554 syphilis cases were reported in this province. Concurrently, syphilis spatial clustering was found in the city of Shenzhen (13). A total of 6,807 syphilis cases were reported in 2013 in Shenzhen (14). The burden of syphilis in Shenzhen is large, and syphilis control should therefore be regarded as a public health priority.

Although some researchers have reported on seroconversion and its determinants after treatment (15-17), studies on syphilis seronegative conversion and associated factors in Shenzhen are limited. Generally, patients with effective treatment for syphilis have been defined as patients having a $\geq 4$-fold decreased titer after treatment during follow-up. However, some patients do not exhibit seronegative conversion but rather enter a serofast state. Although seroconversion from positive to negative is recognized as the final goal of treatment for doctors and patients, some studies have found that a serofast state can also reduce the psychological burden of patients. Seronegative conversion means that syphilis has been cured completely, without risk of recurrence; thus, it is an important index of effective treatment. Therefore, this study was conducted to explore post-syphilis seronegative conversion predictors. The results can facilitate the identification of high-risk populations

Abbreviations: STI, sexually transmitted infection; TRUST, toluidine red unheated serum test; HR, hazard ratio; CI, confidence interval; STD, sexually transmitted disease; TPPA, Treponema pallidum particle agglutination; BPG, benzathine penicillin G; RPR, rapid plasma reagin. and promote treatment outcomes. Moreover, they can provide a scientific basis for the centralized management of syphilis. In April 2011, Nanshan District, Shenzhen, launched the Syphilis Convergence Case-Management Program to consolidate prevention, treatment, and management for better syphilis control. All health organizations in the district were asked to refer patients with syphilis seropositivity to the STD clinic of the Department of Dermatology and Venereology in Nanshan Center for Chronic Disease Control, where patients were subject to centralized management, standardized treatment, and regular serologic follow-up. By running this program, we aimed to observe the outcome of seronegative conversion after the centralized management of patients with syphilis and to explore potentially associated factors.

\section{MATERIALS AND METHODS}

\section{Study Population}

A retrospective population-based cohort study was conducted. We retrospectively analyzed the data from syphilis patients managed in the clinic of Shenzhen Nanshan Hospital for Chronic Diseases between 2011 and 2019. Patients who met the following criteria were included in the study: (1) diagnosed with syphilis, (2) had related information about treatment, and (3) completed at least one serological follow-up visit after treatment. People with any of the following conditions diagnosed by a doctor were excluded: severe heart, liver, or kidney disease; malignant disease; chronic infectious disease, such as tuberculosis, leprosy, or viral hepatitis; systemic autoimmune disease, such as lupus erythematosus, rheumatoid arthritis, or dermatomyositis; and severe mental illness.

\section{Diagnosis, Treatment, and Follow-Up}

The patients were diagnosed with syphilis infection according to epidemiological history, clinical signs and symptoms, and toluidine red unheated serum test (TRUST)/Treponema pallidum particle agglutination (TPPA) results. Some patients actively underwent syphilis testing when they had venereal symptoms or had engaged in high-risk behavior. Syphilis infection in other patients was discovered through passive testing, such as physical examinations, blood donation and preoperative examination. Non-penicillin-allergic participants were treated with benzathine penicillin G (BPG) in one or two courses (2.4 million units BPG weekly for 2 weeks as one course). Penicillin-allergic participants received doxycycline $(100 \mathrm{mg}$ taken orally twice daily for 14 days). All participants were notified of the need for follow-up for 1 year after treatment. Quantitative and qualitative testing of TRUST, indicating serological conversion negativity or lack thereof, was performed during follow-up, and the resolution of clinical signs and symptoms was evaluated.

\section{Data Collection}

Qualified professional STD physicians were installed as program investigators to collect data. They underwent unified training and became familiar with a written investigation manual prior to data collection. Face-to-face interviews were conducted with a designed questionnaire to obtain related information from the 
patients at the beginning of the study. Written patient consent was obtained according to institutional guidelines.

\section{Laboratory Testing}

Blood specimens were tested for syphilis. TRUST (Rongsheng Biotech Company, Shanghai, China) and TPPA tests (Fujirebio, Tokyo, Japan) were performed on serum samples. The operational instructions were strictly obeyed, and the outcomes were identified by multiple researchers.

\section{Statistical Analysis}

The information was recorded in a database using EpiData 3.0 and analyzed using SPSS 25.0. Descriptive statistics and chisquare tests were used to analyze the differences in seronegative conversion of different syphilis subpopulations. The endpoint was defined as the outcome of the TRUST test becoming negative. If the endpoint was not reached, the patient was defined as censored. If someone was lost to follow-up, the final followup result was used. Univariable and multiple Cox proportional hazard analyses were performed to determine the factors associated with seronegative conversion among the patients with syphilis. Variables in the univariable analyses with a $p$-value $<0.30$ were included in subsequent multivariable regression models. Multiple regression models were built using stepwise techniques. The selection of variables in the final model was conducted using a forward-conditional method, with significance levels of $\leq 0.05$ for inclusion and $\geq 0.1$ for exclusion. A $p$-value $\leq 0.05$ was considered statistically significant.

\section{RESULTS}

\section{Characteristics of the Patients}

During the study period, 1,545 patients with syphilis received routine syphilis management; 290 were excluded due to missing follow-up data. Finally, a total of 1,255 eligible patients with syphilis were included. The average observation time was 9.5 \pm 3.2 months. A total of 750 (59.8\%) and 505 (40.2\%) patients were male and female, respectively, corresponding to a sex ratio of 1.5:1. The mean age was $35.9 \pm 11.9$ years, and the ages ranged from 15 to 89 years. There were 490 (39.0\%) unmarried patients, $673(53.6 \%)$ married patients, and 92 (7.3\%) patients who were divorced or widowed. There were 651 (51.9\%) patients with latent syphilis, $113(9.0 \%)$ patients with primary syphilis, 249 (19.8\%) patients with secondary syphilis, and 242 (19.3\%) patients receiving adequate treatment.

\section{Outcomes of Seronegative Conversion Among Syphilis Patients}

Table 1 shows the outcome of seronegative conversion among the patients. According to the TRUST results, the total percentage of seronegative conversions among the patients was $27.6 \%$ (346/1255).

At the end of the study, 257 (34.3\%) male patients with syphilis tested negative, which was more than the number of female patients $(n=89,17.6 \%)(p<0.01)$. Patients who adopted the syphilis test actively showed a higher frequency of TRUST
TABLE 1 | Outcomes of TRUST seronegative conversion among syphilis patients.

\begin{tabular}{|c|c|c|c|c|}
\hline & $\mathbf{N}$ & $\begin{array}{l}\text { TRUST seronegative } \\
\text { conversion }(\mathrm{n}, \%)\end{array}$ & $x^{2}$ & $P$ \\
\hline Age (years) & & & 2.605 & 0.272 \\
\hline $15 \sim 19$ & 32 & $12(37.5)$ & & \\
\hline 20 29 & 427 & $124(29.0)$ & & \\
\hline$\geq 30$ & 796 & $210(26.4)$ & & \\
\hline Sex & & & 41.861 & $<0.01$ \\
\hline Male & 750 & 257 (34.3) & & \\
\hline Female & 505 & 89 (17.6) & & \\
\hline Marital status & & & 4.966 & 0.084 \\
\hline Unmarried & 490 & $152(31.0)$ & & \\
\hline Married & 673 & $169(25.1)$ & & \\
\hline Divorced/Widowed & 92 & $25(27.2)$ & & \\
\hline Method of syphilis discovery & & & 22.226 & $<0.01$ \\
\hline Active detection & 897 & $281(31.3)$ & & \\
\hline Passive detection & 358 & $65(18.2)$ & & \\
\hline Initial TRUST titer & & & 5.253 & 0.022 \\
\hline$\leq 1: 8$ & 711 & $214(30.1)$ & & \\
\hline$>1: 8$ & 544 & $132(24.3)$ & & \\
\hline Syphilis stage & & & 178.673 & $<0.01$ \\
\hline Latent & 651 & 136 (20.9) & & \\
\hline Primary & 113 & $84(74.3)$ & & \\
\hline Secondary & 249 & $95(38.2)$ & & \\
\hline After adequate treatment & 242 & $31(12.8)$ & & \\
\hline Treatment regimen & & & 18.776 & $<0.01$ \\
\hline Untreated & 159 & $22(13.8)$ & & \\
\hline One course of BPG & 928 & 281 (30.3) & & \\
\hline Two courses of BPG & 134 & $34(25.4)$ & & \\
\hline $\begin{array}{l}\text { Replacement therapy with } \\
\text { doxycycline }\end{array}$ & 34 & $9(26.5)$ & & \\
\hline People living with HIV & & & 1.978 & 0.159 \\
\hline Yes & 148 & $48(32.4)$ & & \\
\hline No & 1,107 & 298 (26.9) & & \\
\hline Male homosexuality & & & 3.361 & 0.067 \\
\hline Yes & 174 & 58 (33.3) & & \\
\hline No & 1,081 & 288 (26.6) & & \\
\hline Bisexuality & & & & $<0.01$ \\
\hline Yes & 41 & $22(53.7)$ & & \\
\hline No & 1,214 & $324(26.7)$ & & \\
\hline
\end{tabular}

seroconversion from positive to negative than those who received the test passively ( $n=281,31.3 \%$ vs. $n=65,18.2 \%, p<0.01$ ).

Patients with a baseline TRUST titer of $\leq 1: 8$ had a higher frequency of seronegative conversion than those with a TRUST titer of $>1: 8(n=214,30.1 \%$ vs. $n=132,24.3 \%, p=0.022)$. Eighty-four $(74.3 \%)$ patients with primary syphilis underwent TRUST seronegative conversion, with a higher frequency of conversion observed in these patients than in patients with secondary syphilis $(n=95,38.2 \%)$, patients with latent syphilis ( $n=136,20.9 \%)$, and patients who were receiving adequate treatment $(n=31,12.8 \%)(p<0.01)$. Patients with one course of BPG had a higher frequency of TRUST seronegative conversion 
( $n=281,30.3 \%)$ than those with no treatment $(n=22$, $13.8 \%)$, two courses of BPG $(n=34,25.4 \%)$, or replacement therapy with doxycycline $(n=9,26.5 \%)$. A higher frequency of TRUST seronegative conversion was found in bisexual patients with syphilis $(n=22,53.7 \%)$ than in heterosexual patients $(p$ $<0.01)$. TRUST seronegative conversion for syphilis was not significantly associated with age, marital status, HIV infection, or male homosexuality $(p>0.05)$.

\section{Factors Associated With Seronegative Conversion Among Syphilis Patients}

Univariable Cox proportional hazard regression analyses were performed to identify the factors associated with seronegative conversion. Male patients with syphilis were more likely to test seronegative than were female patients $(\mathrm{HR}=1.88,95 \% \mathrm{CI}=$ $1.48-2.39 ; p<0.01)$. Patients who had actively undergone the syphilis test were more likely to show seronegative conversion than those who had undergone the test passively $(\mathrm{HR}=$ $1.61,95 \% \mathrm{CI}=1.23-2.11, p<0.01$ ). Furthermore, patients with a lower initial TRUST titer of $\leq 1: 8$ were more likely to test negative than those with an initial TRUST titer of $>1: 8(\mathrm{HR}=1.34,95 \% \mathrm{CI}=1.08-1.67, p=0.01)$. Compared to patients who were receiving adequate treatment, patients with latent syphilis $(\mathrm{HR}=1.84,95 \% \mathrm{CI}=1.24-2.71, p<$ 0.01 ), primary syphilis $(\mathrm{HR}=7.60,95 \% \mathrm{CI}=5.03-11.48$, $p<0.01)$, and secondary syphilis $(\mathrm{HR}=3.18,95 \% \mathrm{CI}=$ 2.12-4.76, $p<0.01$ ) were more likely to show seronegative conversion. Compared to untreated patients, those receiving treatment regimens, including one course of $\mathrm{BPG}(\mathrm{HR}=2.38$, 95\% CI $=1.54-3.67, p<0.01)$, two courses of BPG $(\mathrm{HR}=$ $1.84,95 \% \mathrm{CI}=1.08-3.14, p=0.03)$, and replacement therapy with doxycycline $(\mathrm{HR}=2.26,95 \% \mathrm{CI}=1.04-4.90, p=0.04)$, were more likely to test negative. Male homosexual individuals were more likely to show seronegative conversion $(\mathrm{HR}=1.36$, 95\% CI $=1.03-1.80, p=0.03$ ) and bisexual syphilis patients $(\mathrm{HR}=1.71,95 \% \mathrm{CI}=1.11-2.64, p=0.01)$ were associated with syphilis TRUST seroconversion from positive to negative (Table 2).

All variables at $p<0.3$ in the univariable analyses were included in the multivariable Cox regression analysis. A forward selection (conditional) method was conducted to select variables, with a significance level of $\leq 0.05$ required for inclusion and of $\geq 0.1$ required for exclusion. The multivariable analysis suggested that patients of younger age (15-19 years vs. $\geq 30$ years: HR $=2.18,95 \% \mathrm{CI}=1.21-3.94, p=0.01)$ and male gender $(\mathrm{HR}$ $=1.45,95 \% \mathrm{CI}=1.12-1.88, p=0.01$ ) were more likely to test negative. Patients with a lower initial TRUST titer of $\leq 1: 8$ more easily underwent TRUST seroconversion from positive to negative than those with a TRUST titer of $>1: 8(\mathrm{HR}=2.23$, $95 \% \mathrm{CI}=1.72-2.88$ ). Moreover, compared to patients who were receiving adequate treatment, patients at different disease stages, including latent syphilis $(\mathrm{HR}=1.98,95 \% \mathrm{CI}=1.34-2.93, p$ $<0.01)$, primary syphilis $(\mathrm{HR}=7.67,95 \% \mathrm{CI}=5.01-11.74, p$ $<0.01)$, and secondary syphilis $(\mathrm{HR}=4.83,95 \% \mathrm{CI}=3.09-$ 7.54), were significantly more likely to show syphilis TRUST seroconversion (Table 2).

\section{DISCUSSION}

Syphilis is a persistent public health issue in many countries. Its prevalence has recently increased in some countries $(18,19)$. In Guangdong Province, the syphilis incidence rate increased yearly between 2005 and 2014-from 21.08/100,000 to $52.55 / 100,000$ over this period (20). A systematic review found that $90 \%$ of syphilis patients were from resourcelimited countries (21). Therefore, the Syphilis Convergence Case-Management Project was implemented to promote prevention and control. Through our research, the state of seronegative conversion and a few significant factors associated with seronegative syphilis conversion were discovered. The research results provide some basis for building an effective and synthetic model of syphilis prevention and control.

Only $27.6 \%$ of the patients showed TRUST seronegative conversion at the end of our study. A previous study reported that the rate of syphilis serological cure $(17,22)$ was $65-$ $79 \%$; however, the sample size was smaller than that in the present study. Moreover, some of the patients in our study originated from other health care facilities, and their past medical history and treatments were comparatively complicated. In addition, treatment cost, treatment adherence, and type of health care system might be correlated with seroconversion. The percentage of seronegative conversion in our study also suggests that the level of management of syphilis convergence cases, such as treatment, follow-up, and data collection, needs to be strengthened in the future.

In our study, we found that seronegative conversion was independently associated with younger age, as was also reported by Seña et al. (17), who revealed that the probability of achieving serological cure decreased with age. This association may be related to the senescence of the immune system at older age, which can be expected to influence the serological response to syphilis therapy (22). In addition, more male patients tested negative for TRUST than female patients $(34.3 \%$ vs. $17.9 \%)$. The multivariable analysis also showed that male patients with syphilis were more likely to test seronegative. This difference might be related to the differences in the immune system between men and women (23), which influence the serological response. The exact mechanism underlying the association with sex is still unclear and needs further investigation.

Understanding the relationship of quantitative nontreponemal titers with disease is vital for evaluating treatment response, which can reflect the activity of the disease process or the immune response. Generally, non-treponemal antibody titers are related to disease activity. However, some studies demonstrated that patients with higher baseline rapid plasma reagin (RPR) titers were more likely to achieve serological cure $(17,22)$. Baker-Zander et al. (24) found that Venereal Disease Research Laboratory (VDRL)-immunized rabbits exhibited partial protection against reinfection with $T$. pallidum, which meant that high VDRL antibody titers might help control the infection. However, in our study, the patients with lower initial TRUST titers easily became seronegative. The multivariable 
TABLE 2 | Results of univariable and multivariable analyses of factors associated with TRUST seronegative conversion among syphilis patients.

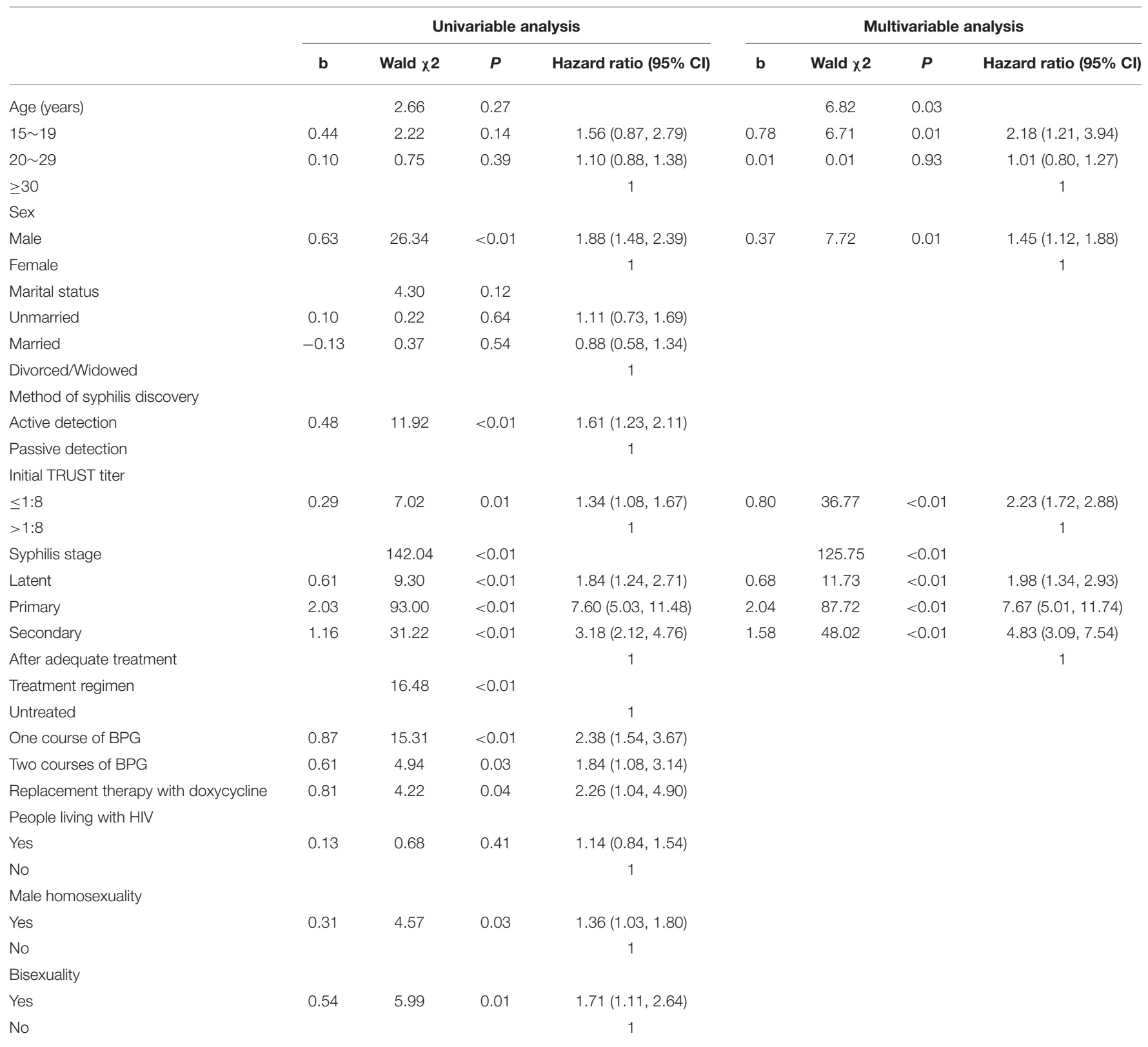

model also revealed that the likelihood of TRUST seronegative conversion was increased with a decrease in the initial TRUST titer. There may be a negative association between the TRUST titer and the quantity of T. pallidum. The detailed mechanism requires clarification through further studies. Hence, the level of syphilis antibody titer might correspond to the levels of syphilis and immune response. Facilitating routine syphilis serological tests and follow-up in the clinic is integral to observing the efficacy of treatment and preventing adverse outcomes.

We also found that patients with primary syphilis were more likely to experience TRUST seronegative conversion than those who were receiving adequate treatment before enrollment and more so than patients with secondary and latent syphilis. The results of the current study were consistent with a previous observation that patients with secondary syphilis were more likely to experience treatment failure than those with primary syphilis (25). In addition, Tong et al. reported that the rate of serological cure decreased in the order of primary, secondary, latent, and tertiary syphilis (22). The differences in immunological functions among different disease stages may be responsible for this observation. These results suggest that enhancing early diagnosis, treatment, and city-specific syphilis screening strategies is very important and that the clinical stages of syphilis often overlap. 


\section{LIMITATIONS}

Our study has several limitations. First, there may be some selection bias because several patients were excluded due to missing data. Second, the results correspond to only one district and might not be generalizable to other areas; thus, the representativeness of the sample needs to be improved. Third, some reporting bias may have been inherent in this study because some subjects may have hidden sensitive information.

\section{CONCLUSION}

The present study showed that syphilis TRUST seronegative conversion after treatment was poor. However, syphilis seroconversion from positive to negative was significantly associated with sex, baseline TRUST titer, and disease stage.

\section{DATA AVAILABILITY STATEMENT}

The raw data supporting the conclusions of this article will be made available by the authors, without undue reservation.

\section{ETHICS STATEMENT}

The studies involving human participants were reviewed and approved by Shenzhen Nanshan Center for Chronic Disease

\section{REFERENCES}

1. Wang Q, Liu JZ, Xu JH. Sexually Transmitted Diseases Clinic Prevention and Treatment Guidelines. 1st ed. Shanghai: Shanghai Science and Technology Press (2014)

2. Golden MR, Marra CM, Holmes KK. Update on syphilis: resurgence of an old problem. JAMA. (2003) 290:1510-4 doi: 10.1001/jama.290.11.1510

3. World Health Organization. Global Incidence and Prevalence of Selected Curable Sexually Transmitted Infections - 2008. World Health Organization (2012). Available online at: https://apps.who.int/iris/handle/10665/75181

4. Kularatne RS, Niit R, Rowley J, Kufa-Chakezha T, Peters RPH, Taylor $\mathrm{MM}$, et al. Adult gonorrhea, chlamydia and syphilis prevalence, incidence, treatment and syndromic case reporting in South Africa: estimates using the Spectrum-STI model, 1990-2017. PLoS ONE. (2018) 13:e0205863. doi: 10.1371/journal.pone. 0205863

5. Kidd SE, Grey JA, Torrone EA, Weinstock HSGJ, Torrone EA, Weinstock HS. Increased methamphetamine, injection drug, and heroin use among women and heterosexual men with primary and secondary syphilisUnited States, 2013-2017. MMWR Morb Mortal Wkly Rep. (2019) 68:144-8. doi: 10.15585/mmwr.mm6806a4

6. Bailey H, Turkova A, Thorne C. Syphilis, hepatitis C and HIV in Eastern Europe. Curr Opin Infect Dis. (2017) 30:93-100. doi: 10.1097/QCO.0000000000000326

7. Chen ZQ, Zhang GC, Gong XD, Lin C, Gao X, Liang GJ, et al. Syphilis in China: results of a national surveillance programme. Lancet. (2007) 369:1328. doi: 10.1016/S0140-6736(07)60074-9

8. Yang S, Wu J, Ding C, Cui Y, Zhou Y, Li Y, et al. Epidemiological features of and changes in incidence of infectious diseases in China in the first decade after the SARS outbreak: an observational trend study. Lancet Infect Dis. (2017) 17:716-25. doi: 10.1016/S1473-3099(17) 30227-X

9. Wu Z, Zhou P. Syphilis and social upheaval in China. N Engl J Med. (2010) 363:1088. doi: 10.1056/NEJMc1006525
Control. Written informed consent to participate in this study was provided by the participants' legal guardian/next of kin.

\section{AUTHOR CONTRIBUTIONS}

ZL and BL conceived and designed the study. JY and QW implemented the study and conducted data collection. LT and LZ contributed to data analysis. ZL and YD wrote and drafted the manuscript. JM was involved in critical revision of the manuscript. All authors have read and approved the final manuscript.

\section{FUNDING}

The Shenzhen Healthcare Research Project (SZGW2018001) supported this study.

\section{ACKNOWLEDGMENTS}

We thank the patients for their cooperation in this study as well as all the individuals who contributed to this work. We also thank the Shenzhen Healthcare Research Project for supporting our research and Editage for English language editing.
10. Tucker JD, Cohen MS. China's syphilis epidemic: epidemiology, proximate determinants of spread, and control responses. Curr Opin Infect Dis. (2011) 24:50-5. doi: 10.1097/QCO.0b013e32834204bf

11. Lin CC, Gao X, Chen XS, Chen Q, Cohen MS. China's syphilis epidemic: a systematic review of seroprevalence studies. Sex Transm Dis. (2006) 33:72636. doi: 10.1097/01.olq.0000222703.12018.58

12. Yang LG, Tucker JD, Yang B, Shen SY, Sun XF, Chen YF, et al. Primary syphilis cases in Guangdong Province 1995-2008: opportunities for linking syphilis control and regional development. BMC Public Health. (2010) 10:793. doi: 10.1186/1471-2458-10-793

13. Wong NS, Chen L, Tucker JD, Zhao P, Goh BT, Poon CM, et al. Distribution of reported syphilis cases in South China: spatiotemporal analysis [Sci. rep.:9090]. Sci Rep. (2018) 8:9090. doi: 10.1038/s41598-018-27173-y

14. Lan L, Wu X, Zhang C, Hong F. Epidemiological analysis of syphilis in Shenzhen from 2004 to 2013. China Trop Med. (2015) 15:700-3. doi: 10.13604/j.cnki.46-1064/r.2015.06.018

15. Xu JJ, Zhang M, Brown K, Reilly K, Wang H, Hu Q, et al. Syphilis and HIV seroconversion among a 12-month prospective cohort of men who have sex with men in Shenyang, China. Sex Transm Dis. (2010) 37:432-9. doi: 10.1097/OLQ.0b013e3181d13eed

16. Zou X, Ling L, Zhang L. Trends and risk factors for HIV, HCV and syphilis seroconversion among drug users in a methadone maintenance treatment programme in China: a 7-year retrospective cohort study. BMJ Open. (2015) 5:e008162. doi: 10.1136/bmjopen-2015-008162

17. Seña AC, Wolff M, Martin DH, Behets F, Van Damme K, Leone P, et al. Predictors of serological cure and Serofast State after treatment in HIVnegative persons with early syphilis. Clin Infect Dis. (2011) 53:1092-9. doi: 10.1093/cid/cir671

18. Fenton KA, Breban R, Vardavas R, Okano JT, Martin T, Aral S, et al. Infectious syphilis in high-income settings in the 21st century. Lancet Infect Dis. (2008) 8:244-53. doi: 10.1016/S1473-3099(08)70065-3

19. Mutagoma M, Remera E, Sebuhoro D, Kanters S, Riedel DJ, Nsanzimana S. The prevalence of syphilis infection and its associated factors in the general 
population of Rwanda: a national household-based survey. J Sex Transm Dis. (2016) 2016:4980417. doi: 10.1155/2016/4980417

20. Zou Y, Liu F, Chen L, Shen HC, Huang SJ, Zheng HP, et al. Epidemiological trend and disease burden of syphilis in Guangdong Province, 2005-2014. J Sun Yat-Sen Univ Med Sci. (2016) 37:142-7. doi: 10.13471/j.cnki.j.sun.yat-sen.univ(med.sci).2016.0025

21. Tucker JD, Bu J, Brown LB, Yin YP, Chen XS, Cohen MS. Accelerating worldwide syphilis screening through rapid testing: a systematic review. Lancet Infect Dis. (2010) 10:381-6. doi: 10.1016/S1473-3099(10)7 0092-X

22. Tong ML, Lin LR, Liu GL, Zhang HL, Zeng YL, Zheng WH, et al. Factors associated with serological cure and the serofast state of HIV-negative patients with primary, secondary, latent, and tertiary syphilis. PLoS ONE. (2013) 8:e70102. doi: 10.1371/journal.pone.0070102

23. Whitacre CC, Reingold SC, O'Looney PA, A. gender gap in autoimmunity. Science. (1999) 283:1277-8. doi: 10.1126/science.283.5406.1277

24. Baker-Zander SA, Shaffer JM, Lukehart SA, VDRL antibodies enhance phagocytosis of Treponema pallidum by macrophages. J Infect Dis. (1993) 167:1100-5. doi: 10.1093/infdis/167.5.1100

25. Luo Z, Zhu L, Ding Y, Yuan J, Li W, Wu Q, et al. Factors associated with syphilis treatment failure and reinfection: a longitudinal cohort study in Shenzhen, ChinaChina. BMC Infect Dis. (2017) 17:620. doi: 10.1186/s12879-017-2715-Z

Conflict of Interest: The authors declare that the research was conducted in the absence of any commercial or financial relationships that could be construed as a potential conflict of interest.

Publisher's Note: All claims expressed in this article are solely those of the authors and do not necessarily represent those of their affiliated organizations, or those of the publisher, the editors and the reviewers. Any product that may be evaluated in this article, or claim that may be made by its manufacturer, is not guaranteed or endorsed by the publisher.

Copyright () 2021 Luo, Ding, Yuan, Wu, Tian, Zhang, Li and Mou. This is an open-access article distributed under the terms of the Creative Commons Attribution License (CC BY). The use, distribution or reproduction in other forums is permitted, provided the original author(s) and the copyright owner(s) are credited and that the original publication in this journal is cited, in accordance with accepted academic practice. No use, distribution or reproduction is permitted which does not comply with these terms. 